\title{
Age and distraction are determinants of performance on a novel visual search task in aged Beagle dogs
}

\author{
Shikha Snigdha • Lori-Ann Christie • \\ Christina De Rivera • Joseph A. Araujo • \\ Norton W. Milgram • Carl W. Cotman
}

Received: 4 November 2010/Accepted: 6 February 2011 /Published online: 19 February 2011

(C) The Author(s) 2011. This article is published with open access at Springerlink.com

\begin{abstract}
Aging has been shown to disrupt performance on tasks that require intact visual search and discrimination abilities in human studies. The goal of the present study was to determine if canines show age-related decline in their ability to perform a novel simultaneous visual search task. Three groups of canines were included: a young group $(N=10 ; 3$ to 4.5 years), an old group ( $N=10 ; 8$ to 9.5 years), and a senior group ( $N=8 ; 11$ to 15.3 years). Subjects were first tested for their ability to learn a simple twochoice discrimination task, followed by the visual search task. Attentional demands in the task were manipulated by varying the number of distracter items; dogs received an equal number of trials with either zero, one, two, or three distracters. Performance on the two-choice discrimination task varied with age, with senior canines making significantly more errors than the young. Performance accuracy on the visual
\end{abstract}

S. Snigdha $(\triangle) \cdot$ L.-A. Christie $\cdot$ C. W. Cotman Institute for Memory Impairments and Neurological Disorders, University of California, Irvine, 1226 Gillespie NRF, Irvine, CA 92697-4540, USA

e-mail: ssnigdha@uci.edu

C. De Rivera • J. A. Araujo • N. W. Milgram

CanCog Technologies Inc.,

Toronto, Canada

C. De Rivera · J. A. Araujo • N. W. Milgram

Department of Pharmacology, University of Toronto,

Toronto, Canada search task also varied with age; senior animals were significantly impaired compared to both the young and old, and old canines were intermediate in performance between young and senior. Accuracy decreased significantly with added distracters in all age groups. These results suggest that aging impairs the ability of canines to discriminate between taskrelevant and -irrelevant stimuli. This is likely to be derived from impairments in cognitive domains such as visual memory and learning and selective attention.

Keywords Aging - Canine - Attention - Distraction · Visual learning

\section{Introduction}

Selective visual attention and learning are particularly sensitive to aging. A common method widely used to research these domains is visual search. This technique requires participants to attend to a target stimulus while disregarding irrelevant "distracter" information (Julesz and Schumer 1981). The effect of the distracter can be evaluated using either serial or simultaneous presentations, processes which are referred to differently by various groups (attentive vs. preattentive, effortful vs. automatic, respectively; for review, see Ball et al. 1988). However, primarily, they refer to sequential or simultaneous presentation and processing of visual stimuli during the test. Serial processing requires more effort or attention and 
includes components of working memory and attention, as the visual stimuli must be stored in memory, albeit briefly. Simultaneous processing on the other hand requires parallel processing of changing visual stimuli. However, since the stimulus is always present in the visual field, no working memory component is involved, and the stimulus is processed preattentively (Ball et al. 1988).

Deficits in both serial and simultaneous processing of visual stimuli increase with age. This agedependent deficit may be linked to decreases in visual processing speed, reduced cognitive resources (as seen by impairments in other cognitive and learning abilities), and inability to ignore distracting information (as observed by decrease performance accuracy in making a correct choice) when presented with increased number of distracters (Baddeley et al. 2001; Costello et al. 2010; Lavie 1995).

Behavioral studies in humans and monkeys have highlighted that the effectiveness of visual selection processes can vary as a function of perceptual object dimensions (Roelfsema 1998; also reviewed in (Yantis and Egeth 1999)). We have previously replicated these functions in a canine model of aging. Performance accuracy declined with increased similarity of the distracter and target stimuli in three-choice (or "oddity") discrimination. In this task, the size, shape, and color (of the positive and negative stimuli) become progressively more similar, resulting in an increase in trials required to reach criterion performance coupled with a decrease in accuracy. This effect was more robust in old dogs when compared to young dogs.

In human studies, decreased prefrontal metabolism and volume are associated with impairment in several domains of attention function such as set shifting and sustained and selective attention (Brooks et al. 2006; Mostofsky et al. 2002; Serences et al. 2004). These effects are further exacerbated by age (Madden 2007; Madden 2007). However, to the best of our knowledge, no previous studies have assessed age-related performance on a test of visual selective attention in canines.

Thus, the goal of the present study was to develop a novel task to assess selective visual attention in dogs. We also attempted to determine if visual processing speed, reduced cognitive resources, and/ or an inability to ignore distracting information, either separately or in combination, was central to driving the attention decline
After initial training on a two-choice discrimination task, we tested the ability of young, old, and senior dogs to respond to the target stimulus when it was presented along with zero, one, two, or three identical distracters. We further increased the difficulty of the task by modifying the distracter stimuli and using a distracter with greater physical similarity of the target for the final seven sessions of the task. Studies in human subjects reveal that challenging discrimination ability by using physically similar stimuli taxes attentional resources in older subjects (Scialfa et al. 1998) and in AD patients (Gainotti et al. 2001). Therefore, we predicted that aging would result in decreased response accuracy coupled with increased response latency, and that greater disruption would be seen when there was an increased similarity of distracter to the positive stimulus.

\section{Materials and methods}

Subjects

A total of 28 beagles were included in the study in three age groups: a young group $(N=10 ; 3$ to 4.5 years; $N=4$ males), an old group ( $N=10 ; 8$ to 9.5 years; $N=5$ males), and a senior group $(N=8 ; 11$ to 15.3 years; $N=2$ males).

Dogs were obtained from CanCog Technologies Inc. (Toronto, Canada) and had identical cognitive testing experience, which included pre-training, as described previously (Milgram et al. 1994), and training on a spatial memory task, the delayed nonmatching to position (DNMP). Inclusion in the study required consistent responsiveness and successful acquisition of the pre-training protocol and the DNMP task. Veterinary examinations were conducted on all dogs prior to the start of the study to assess their general health and to ensure that visual, auditory, and motor functioning were not compromised.

Dogs were group housed and had free access to water. They were fed a standard adult maintenance diet once daily (Purina Pro Plan ${ }^{\circledR}$ Chicken and Rice) so as to maintain a consistent body weight and appropriate body condition, which was assessed every 2 weeks. Housing temperature was held at $21 \pm 6^{\circ} \mathrm{C}$, and relative humidity levels ranged between $15 \%$ and $75 \%$. The study was approved by the CanCog Local Animal Care Committee, and the facility was 
accredited by Ontario Ministry of Agriculture, Farms and Rural Affairs.

Experimental design

Following the completion of a standard pre-training protocol and DNMP task, dogs were tested on the attention task, which consisted of three phases. In phase I, dogs were administered a two-choice object discrimination task. Phases II and III consisted of 14 days of attention testing; the dogs' ability to visually search for and select the stimulus that was rewarded in phase I when it was presented along with zero, one, two, or three distracter stimuli was tested at two difficulty levels. Phase II used the same distracter as used in phase I (i.e., negative object), and phase III used a different distracter that was more similar to the rewarded stimulus, thus increasing the difficulty of the task. For all phases, animals were cognitively tested 7 days a week.

\section{Cognitive testing}

All cognitive testing was conducted in a canine adaptation of the Wisconsin general testing apparatus, as described previously (Milgram et al. 1994). Briefly, the apparatus consisted of a large holding area where the dog was housed during testing, which was separated from the experimenter by a wooden screen containing a one-way mirror and a hinged door at the bottom. A plastic stimulus tray containing either three (for phase I) or four (for phases II and III) food wells was pushed through the hinged door by the experimenter so that the dog could access the stimuli and food rewards by sticking its head through adjustable stainless steel gates at the front of the holding area. The tray was removed out of sight during inter-trial intervals. A dedicated computer program (DogCog(C), CanCog Technologies Inc.) was used to time trials and collect response and latency data during cognitive testing.

Food reward for correct responses during cognitive testing consisted of approximately $1 \mathrm{~g}$ of wet $\mathrm{dog}$ food. To mask the presence of the food reward in the negative food wells, the undersides of all stimuli were baited with the same food such that, while able to smell it, the animals could not see or eat it. For all tasks, a partial correction procedure was used, in which the dogs were permitted to correct their first error during each test session.

\section{Phase I: two-choice discrimination task}

Dogs were initially trained on a two-choice discrimination problem, which requires them to learn that choosing a particular object is always associated with a food reward, while choosing the other is not. Prior to discrimination testing, a preference test was administered to establish if a preference for either object existed. Ten trials were given, and the number of choices for each stimulus was recorded. The non-preferred stimulus served as the rewarded stimulus for phases I, II, and III. Stimuli for phase I consisted of a double-pronged green toy block and a yellow toy banana.

For phase I, animals were given ten trials per session once daily, with an inter-trial interval of $30 \mathrm{~s}$. An error was counted when the dog displaced the non-rewarded stimulus, and a food reward was provided in the food well when the dog displaced the positive stimulus. In order to pass phase I, dogs had to meet a two-stage set of criteria. For the first stage, the animal had to average at least $80 \%$ correct over two test sessions or at least $90 \%$ on a single session. For the second stage, the dog had to respond correctly on at least $70 \%$ of the trials over three successive sessions. Subjects had a maximum of 20 sessions to attain criterion. After a dog reached the learning criteria or the maximum number of sessions, it was immediately moved onto phase II.

\section{Phases II and III: attention task}

During phases II and III, dogs were tested on the attention task. Seven test sessions were administered during each phase once daily. Each test session consisted of 12 trials with inter-trial intervals of $30 \mathrm{~s}$. During each session, an equal number of trials using zero, one, two, or three negative stimuli (i.e., distracters) were administered.

The same positive and negative stimuli that were used in phase I were used again in phase II. In phase III, the same positive stimulus was used, but a new stimulus that more closely resembled the positive stimulus was used for negative stimuli (i.e., either a single-pronged, blue toy block or a small, yellow toy French fry).

\section{Statistics}

All statistical analyses were conducted using SPSS 15.0 for Windows. The number of errors made on the 
two-choice discrimination task (phase I) was analyzed for age effects using a one-way analysis of variance (ANOVA). For the attention task, proportion of correct trials was analyzed using two-way repeatedmeasures ANOVAs for phases II and III separately, in which the number of distracters served as the withinsubject variable and age group as the between-subject variable. Similarly, response latencies were analyzed using repeated-measures ANOVAs, with number of distracters as the within-subject variable. When statistically significant overall effects were found (i.e., $p \leq 0.05$ ), Bonferroni corrected post hoc tests were conducted to determine the age group and distracter number effects.

\section{Results}

Phase I: two-choice discrimination

One-way ANOVA showed a significant overall effect of age group $(F(2,25)=7.133 ; p-0.004$, Fig. 1) on number of errors made prior to reaching criterion performance or over 20 sessions. As can be seen in Fig. 1, senior dogs made more errors on the twochoice discrimination task, while old dogs were intermediate in performance between the young and senior groups.

Post hoc $t$ tests confirmed that senior dogs differed from young dogs $(p=0.002)$, while old dogs did not

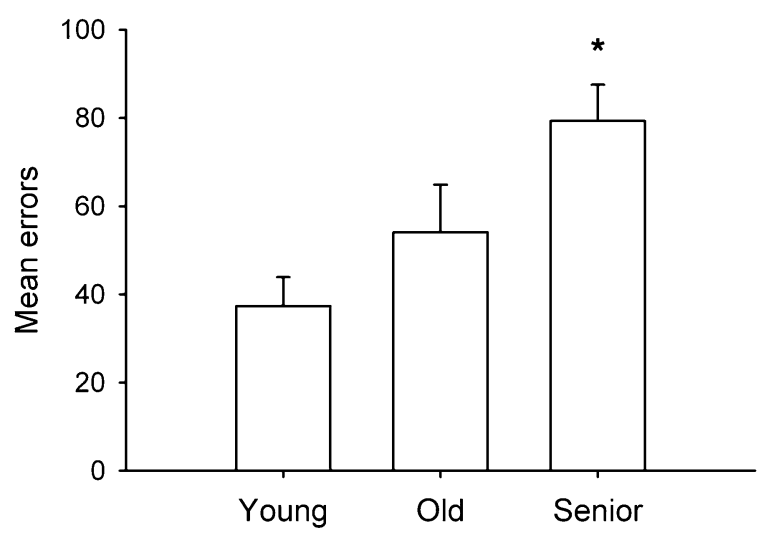

Fig. 1 Two-choice discrimination learning is impaired in senior dogs. Mean errors to acquire the task are shown for each age group. Senior dogs made significantly more errors than young dogs and tended to make more errors compared to old dogs, while the old and young groups did not differ. Data are shown as mean $\pm \mathrm{SEM} * p<0.05$, significant increase in errors compared with young differ significantly from young $(p=0.248)$ or senior animals $(p=0.086)$.

Phases II and III: attention task

In phase II, the same positive and negative (distracter) stimuli were used as in phase I. We assessed whether mean response latency differed between the age groups as a function of the number of distracter items presented. For this analysis, we excluded outlying values (those that were greater than three standard deviations above or below the mean).

A two-way repeated-measures ANOVA using proportion correct trials as the dependent measure showed significant effects of both age group $(F(2,25)=17.2$; $p<0.001)$ and distracter number $(F(2,50)=22.5$; $p<0.001$; see Fig. 2a). The interaction effect was not significant $(F(4,50)=1.02, p=\mathrm{NS})$. Further post hoc $t$ tests showed that performance accuracy in senior dogs was significantly lower compared to both young $(p<0.001)$ and old $(p<0.001)$ animals, while old dogs showed only a trend for decreased accuracy compared to young dogs $(p=0.086)$.

We found a significant effect of distracter number $(F(3,75)=22.09 ; p<0.001)$, but not age $(F(2.75)=$ 9.24; $p=\mathrm{NS}$ ), using a repeated-measures ANOVA, with response latency as the dependent variable (see Fig. 2b). The age by distracter interaction was not significant $(F(6,75)=0.85, p=0.53)$.

A paired $t$ test comparing latencies from the first three sessions versus the last three sessions (across all age groups) was significant $(t(27)=2.247 ; p=0.033)$, with all groups responding more slowly on the first three sessions compared to the last three sessions. This suggested that the excluding values from the first three test sessions would provide a more stable measure of latency. Therefore, response latency was also assessed using a one-way ANOVA across both phases, excluding values from the first three test sessions. We found a significant effect of age if such an analysis was performed $(F(2,25)=3.34 ; p<0.05)$ with young animals showing a significant difference compared to the senior animals $(p<0.05)$. Neither the young versus old $(p=0.282)$ nor old versus senior $(p=0.534)$ comparisons differed significantly.

In phase III, when different distracter stimuli were presented along with the rewarded stimulus, we once again excluded outlying latency values using an identical criterion as in phase II. 

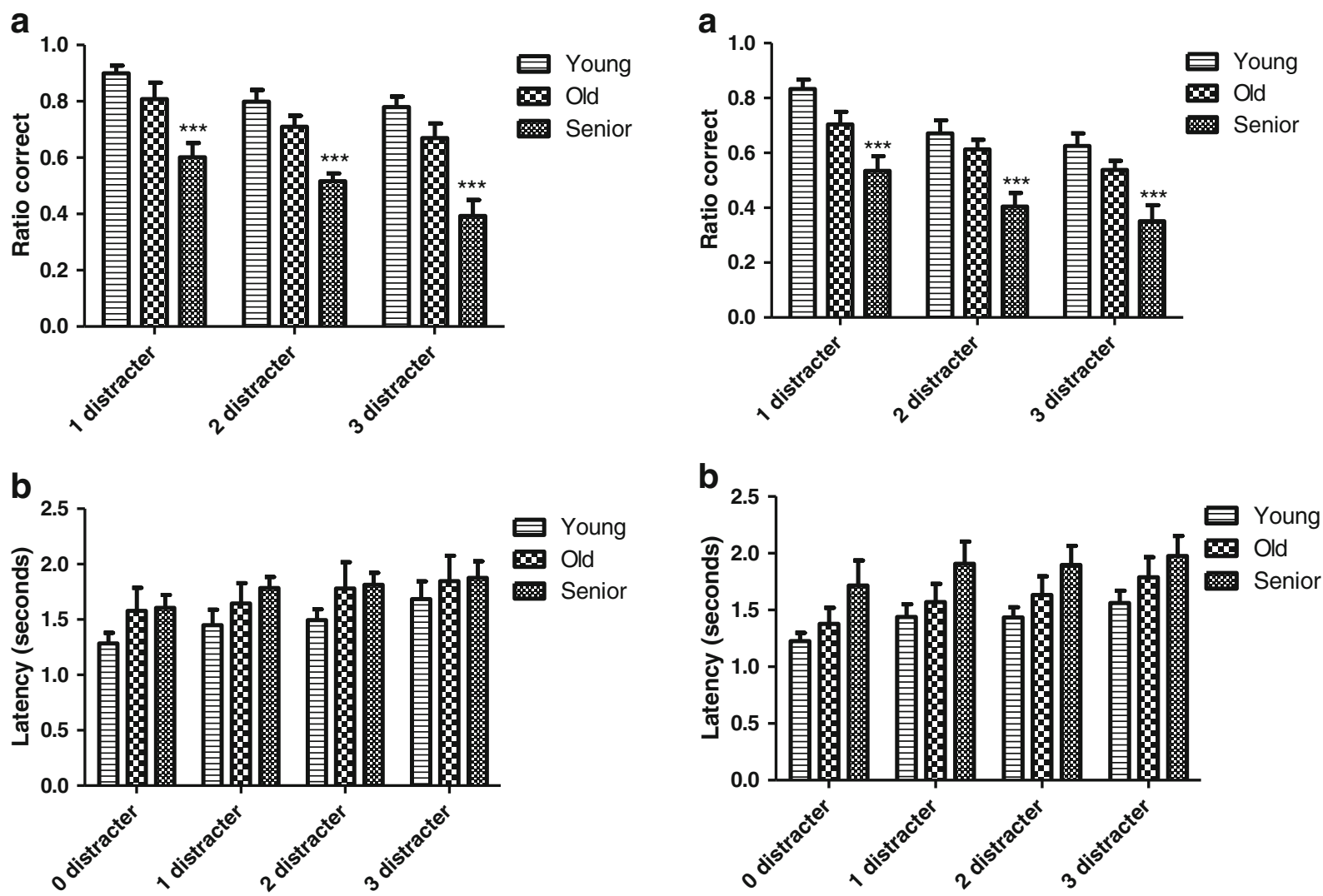

Fig. 2 Performance accuracy shown as ratio correct (a) and response latency shown in seconds (b) across all age groups in phase II using the same stimuli used for the two-choice discrimination task. Senior dogs show significantly decreased accuracy, but not latency, compared to young dogs. Latency increases with increasing number of distracters across all groups. Data are shown as mean \pm SEM. $* * * p<0.001$, significant decrease in ratio of correct response compared with young

Performance accuracy varied with age $(F(2,25)=$ $12.89 ; p<0.001)$ and distracter number $(F(2,50)=$ $36.29 ; p<0.001)$, but the interaction was not significant $(F(4,50)=0.47, p=\mathrm{NS})$. Post hoc tests showed that senior dogs $(p<0.001)$ differed compared to young animals. Although the performance of old dogs was intermediate between senior and young dogs, there was no significant difference when compared to young or old dogs (see Fig. 3a).

In this phase of the task, repeated-measures ANOVA on latency revealed a significant main effect of distracter number $(F(3,75)=34.90, p<0.001)$ but not age $(F(2.75)=2.35 ; p=\mathrm{NS})$. The group interaction effect was also not significant $(F(6,75)=0.78, p=\mathrm{NS}$; see Fig. 3b).

Fig. 3 Performance accuracy shown as ratio correct (a) and response latency shown in seconds (b) across all age groups in phase III using stimuli different from those used for the twochoice discrimination task. Senior dogs show significantly decreased accuracy, but not latency, compared to young dogs. Latency increases with increasing number of distracters across all groups. Data are shown as mean \pm SEM. $* * * p<0.001$, significant decrease in ratio of correct response compared with young

\section{Discussion}

The main aims of this investigation were (1) to determine the effect of aging on selective attention function in dogs and (2) to establish specific factors that underlie the attention deficits. To this end, we hypothesized that reduced cognitive resources, an inability to ignore distracting information and/or reduced visual processing speed, would be three fundamental processes central to driving the decline in attention function.

To assess selective attention, we first trained animals to discriminate between two objects and then tested the animals with the one positive object and 
with zero, one, two, or three replicates of the negative object. The underlying rationale was that the task was essentially a visual search task requiring the subjects to locate the correct object. This hypothesis would predict that the greater the number of replicates of the incorrect objects, the poorer the animals would perform, as reflected by increased reaction time and decrease in ratio of correct response. Results from this investigation show that aging impairs response accuracy in the visual search task described here. The likelihood that three specific factors contribute in part to this impairment is discussed in detail further.

It has been suggested by several groups that selective attention ability depends on cognitive and attentional resources (Groth and Allen 2000; Lavie and Tsal 1994). Older subjects, who have reduced processing resources, have therefore been reported to perform poorly on two-choice visual tasks compared to younger subjects (Madden et al. 1992). Our findings replicate this effect in aged versus young dogs. Senior dogs showed a robust increase in the number of errors on the two-choice discrimination of the task compared to young and old dogs. The outcome measure of the two-choice discrimination task determined the general visual learning ability of the dogs and confirms the hypothesis that aging impairs learning ability in dogs. This finding is consistent with previous findings in the dog (Tapp et al. 2003) and makes a case for increased deficit in the cognitive reserve in aged dogs as compared to younger dogs.

The second factor was the inability to ignore distracting information: Several groups have shown that aging impairs performance in selective attention tasks (Groth and Allen 2000; Nuechterlein et al. 1983; Parasuraman 1979; Parasuraman et al. 2002; Rabbitt 1965). Phases II and III of the task were designed to place a greater emphasis on selective attention and invoked preattentive or simultaneous processing abilities of visual stimuli in the subjects. The level of difficulty was also varied within each phase by changing the number of distracters and by increasing the similarity of distracter to target stimuli. As can be seen from the results, senior dogs were impaired in their ability to ignore distracting information in their field of view.

This is consistent with several results from functional neuroimaging and neurophysiological studies that demonstrate increases in neural activity and neural spiking in response to attended visual events over ignored stimuli in sensory cortical regions (Desimone and Duncan 1995; Moran and Desimone 1985; O'Craven et al. 1997; Reynolds and Desimone 1999). More recently, it was reported that older adults have reduced neural responses to sensory information (Peiffer et al. 2009). Such functional differences in cortical activity between older and younger adults may provide the neural basis for the age-related deficit in ignoring irrelevant information. The effect of distraction in impairing performance in attention-based tasks has been attributed to weakened inhibitory control (Duchek et al. 1998) which has also been described in the dog (Tapp et al. 2003). Moreover, it had been reported, as early as 1965, that reduced inhibitory capacity results in vulnerability of older subjects to distracting stimuli (Rabbitt 1965). In this study, we found a significant decrease in correct response to the target stimulus despite similar reaction times in senior dogs, suggesting that similar to human subjects, older dogs also show inhibitory decrements with age.

Although senior dogs differed significantly from adult dogs on error scores, the response latencies were not significantly different from younger dogs when assessed over all test trials. However, since the response latencies were not stable during the first three trials, we excluded these values from our analyses and found that when measured across the more stable trials, senior dogs take significantly longer to respond. Thus, reaction time, which measured the speed of processing in this test design, was greater for older dogs.

One major difference between our study and the others is the use of 3-s inspection times before allowing the animals to respond to stimulus presentation. This may have reduced the magnitude of difference in response time between groups. Alternatively, it is possible to speculate that the criterion described by Fisk and Schneider (1983) is implicated. The authors suggested that simultaneous processing can be done automatically (preattentively), while serial processes require more processing time. Since this study was designed to test simultaneous and not serial processing, it is possible that the attentional resources of the dogs were not taxed enough to manifest as increase in reaction time across all trials. However, when only the more stable responses were taken into account, a robust effect was observed. Indeed, this is in agreement with the load theory (Lavie 1995) which states that selective attention varies as a function of the load (Greenwood et al. 1997). 
The complex question of age-related decline in visual search ability evidently requires more work to precisely define the processes involved. However, this investigation clearly demonstrates that age-related impairments in selective attention is associated with, and indeed, may be derived from deficits in cognitive resources and an inability to ignore distracting information. It also provides strong evidence for the idea that age-induced deficits in selective attention occur not only in tasks which tap into working memory processes but also in less strenuous tests which require what may possibly be preattentive or automatic processing demands on cognitive resources.

Acknowledgement Funding from the NIH/NIA AG12694 supported this study.

Open Access This article is distributed under the terms of the Creative Commons Attribution Noncommercial License which permits any noncommercial use, distribution, and reproduction in any medium, provided the original author(s) and source are credited.

\section{References}

Baddeley AD, Baddeley HA, Bucks RS, Wilcock GK (2001) Attentional control in Alzheimer's disease. Brain 124:1492-1508

Ball KK, Beard BL, Roenker DL, Miller RL, Griggs DS (1988) Age and visual search: expanding the useful field of view. J Opt Soc Am A 5:2210-2219

Brooks JO 3rd, Wang PW, Strong C, Sachs N, Hoblyn JC, Fenn R, Ketter TA (2006) Preliminary evidence of differential relations between prefrontal cortex metabolism and sustained attention in depressed adults with bipolar disorder and healthy controls. Bipolar Disord 8:248-254

Costello MC, Madden DJ, Mitroff SR, Whiting WL (2010) Age-related decline of visual processing components in change detection. Psychol Aging 25:356-368

Desimone R, Duncan J (1995) Neural mechanisms of selective visual attention. Annu Rev Neurosci 18:193-222

Duchek JM, Hunt L, Ball K, Buckles V, Morris JC (1998) Attention and driving performance in Alzheimer's disease. J Gerontol B Psychol Sci Soc Sci 53:P130-P141

Fisk AD, Schneider W (1983) Category and word search: Generalizing search principles to complex processing. J Exp Psychol: Learning, Memory, and Cognition, 9 (2):177-195

Gainotti G, Marra C, Villa G (2001) A double dissociation between accuracy and time of execution on attentional tasks in Alzheimer's disease and multi-infarct dementia. Brain 124:731-738

Greenwood PM, Parasuraman R, Alexander GE (1997) Controlling the focus of spatial attention during visual search: effects of advanced aging and Alzheimer disease. Neuropsychology 11:3-12
Groth KE, Allen PA (2000) Visual attention and aging. Front Biosci 5:D284-D297

Julesz B, Schumer RA (1981) Early visual perception. Annu Rev Psychol 32:575-627

Lavie N (1995) Perceptual load as a necessary condition for selective attention. J Exp Psychol Hum Percept Perform 21:451-468

Lavie N, Tsal Y (1994) Perceptual load as a major determinant of the locus of selection in visual attention. Percept Psychophys 56:183-197

Madden DJ (2007) Aging and visual attention. Curr Dir Psychol Sci 16:70-74

Madden DJ, Pierce TW, Allen PA (1992) Adult age differences in attentional allocation during memory search. Psychol Aging 7:594-601

Milgram NW, Head E, Weiner E, Thomas E (1994) Cognitive functions and aging in the dog: acquisition of nonspatial visual tasks. Behav Neurosci 108:57-68

Moran J, Desimone R (1985) Selective attention gates visual processing in the extrastriate cortex. Science 229:782784

Mostofsky SH, Cooper KL, Kates WR, Denckla MB, Kaufmann WE (2002) Smaller prefrontal and premotor volumes in boys with attention-deficit/hyperactivity disorder. Biol Psychiatry 52:785-794

Nuechterlein KH, Parasuraman R, Jiang Q (1983) Visual sustained attention: image degradation produces rapid sensitivity decrement over time. Science 220:327-329

O'Craven KM, Rosen BR, Kwong KK, Treisman A, Savoy RL (1997) Voluntary attention modulates fMRI activity in human MT-MST. Neuron 18:591-598

Parasuraman R (1979) Memory load and event rate control sensitivity decrements in sustained attention. Science 205:924-927

Parasuraman R, Greenwood PM, Sunderland T (2002) The apolipoprotein $\mathrm{E}$ gene, attention, and brain function. Neuropsychology 16:254-274

Peiffer AM, Hugenschmidt CE, Maldjian JA, Casanova R, Srikanth R, Hayasaka S, Burdette JH, Kraft RA, Laurienti PJ (2009) Aging and the interaction of sensory cortical function and structure. Hum Brain Mapp 30:228-240

Rabbitt P (1965) An age-decrement in the ability to ignore irrelevant information. J Gerontol 20:233-238

Reynolds JH, Desimone R (1999) The role of neural mechanisms of attention in solving the binding problem. Neuron 24(19-29):111-125

Roelfsema PR (1998) Solutions for the binding problem. Z Naturforsch C 53:691-715

Scialfa CT, Esau SP, Joffe KM (1998) Age, target-distractor similarity, and visual search. Exp Aging Res 24:337358

Serences JT, Schwarzbach J, Courtney SM, Golay X, Yantis S (2004) Control of object-based attention in human cortex. Cereb Cortex 14:1346-1357

Tapp PD, Siwak CT, Estrada J, Head E, Muggenburg BA, Cotman CW, Milgram NW (2003) Size and reversal learning in the beagle dog as a measure of executive function and inhibitory control in aging. Learn Memory 10:64-73

Yantis S, Egeth HE (1999) On the distinction between visual salience and stimulus-driven attentional capture. J Exp Psychol Hum Percept Perform 25:661-676 